\title{
A Fundamental Study of Laser-Induced Breakdown Spectroscopy Using Fiber Optics for Remote Measurements of Trace Metals
}

March 2000

Scott R. Goode, Department of Chemistry and Biochemistry, University of South Carolina, Columbia SC 29208, 803-777-2601, Goode@sc.edu

S. Michael Angel, Department of Chemistry and Biochemistry, University of South Carolina, Columbia SC 29208, 803-777-2601, Angel@mail.chem.sc.edu

This proposal has supported 7 graduate students and/or postdoctoral associates

\section{Research Objectives}

- Develop a fiber-optic imaging probe for microanalysis of solid samples.

- Design a time-resolved plasma imaging system to measure the development of the LIBS signal.

- Setup a laboratory system capable of timing two lasers independently, for optimizing and characterizing dual-pulse LIBS.

- Compare the development of laser-induced plasmas generated with a single laser pulse to the development of laserinduced plasmas generated with a pre-ablation spark prior to sample ablation.

- Examine the effect of sample matrix on the LIBS signals of elements in different sample matrices.

- Investigate the effect of excitation wavelength of the ablation beam in pre-ablation spark dual-pulse LIBS experiments.

- Determine the effect of the physical properties of the sample on the mass of materials ablated.

\section{Research Progress and Implications}

- Developed fiber-optic LIBS and Raman imaging probe for remote analyses.

We developed a unique fiber-optic probe that is capable of acquiring both LIBS and Raman spectra with spatial resolution. This probe was used to demonstrate the first simultaneous measurement of LIBS and Raman spectra and images. The design and implementation of the fiber-optic probe for obtaining LIBS spectra, Raman spectra, and Raman images has been described in Appl. Spectrosc. and a paper in J. Raman Spectroscopy includes results from the Raman portion of the LIBS/Raman microprobe.

- Acquired time-resolved single pulse laser-induced plasma imaging experiments.

We have successfully used time-resolved plasma imaging to measure the development of the LIBS signal.

- Investigated collinear dual-pulse LIBS.

We have fabricated a laboratory system to time two lasers independently for optimizing and characterizing dual-pulse LIBS.

We have successfully produced enhanced emission signals over conventional, single pulse experiments for a number of different samples.

The dual pulse collinear LIBS work has been published in SPIE Proc.

- Developed new pre-ablation dual-pulse LIBS technique for enhanced ablation and emission

We discovered a new dual-pulse LIBS technique that gives enhanced LIBS signals for a large variety of samples and used this new method to obtain large increases in sensitivity for elements in prototypical waste glass samples obtained from the SRTC. A paper was accepted for publication to SPIE Proc. describing the new pre-ablation spark technique with preliminary results obtained, and a paper has been submitted to Appl. Spectrosc. describing the new pre-ablation spark, dual-pulse method discovery and improvements to LIBS analyses using this method. A patent disclosure on the pre-ablation spark, dual-pulse LIBS technique has also been submitted.

- Performed time-resolved pre-ablation spark plasma imaging experiments.

We have begun to compare laser-induced plasmas generated with a single laser pulse to the development of laser-induced plasmas generated with a pre-ablation. These experiments show very large increases in the overall plasma emitting volume for pre-ablation spark dual-pulse plasmas compared to conventional single pulse in experiments for both copper and lead samples.

- Examined the effect of sample matrix on the LIBS signals.

We studied the enhancements of the relatively volatile zinc compared to the less volatile copper in samples of brass while varying the irradiance and it appears that the sample matrix can have a large effect on the extent of signal enhancement. 
- Examined the effect of excitation wavelength of the ablation beam in pre-ablation spark dual-pulse LIBS experiments.

We have begun to look at the effect of changing the laser wavelength used for ablation in the dual-pulse experiments. For all samples we have tested so far there is little or no enhancement when the ultraviolet ablation pulse is used.

\section{- Determined the effect of the physical properties of the sample on the mass of materials ablated.}

Four samples of high purity copper were processed to vary the tensile strength of the samples. Results show that the depth of the crater increases regularly with number of laser pulses and with the tensile strength of the sample at both high and low irradiances. Volumes, and presumably the mass ablated, go through a maximum in samples of intermediate tensile strength The results of the measurements of crater profiles were presented at the Winter Plasma Conference in January, 2000 and have been submitted for publication.

\section{- Developed and tested methods for alloy identification by LIBS.}

We developed methods to analyze the entire spectrum to quickly identify the class of material such as stainless steel, carbon steel, mild steel, brass.... The identification of class of material based on a single LIBS spectrum is $97.4 \%$ accurate, and the correct alloy is identified in $79.9 \%$ of the cases. This work has been described at two national meetings and has been submitted for publication in J. Anal. Atomic Spectrosc.

\section{Planned Activities}

We propose to investigate the new dual-pulse excitation scheme, to use psec laser pulses and to extend the technique to solutions. We will design and test LIBS probes in a cone-penetrometer mockup experiment. These experiments are in progress, and will be substantially complete at the end of the award (March 2001).

\section{Information Access}

\section{Publications:}

B. J. Marquardt, B. M. Cullum, T. J. Shaw and S. M. Angel, "Fiber-optic probe for determining heavy metals in solids based on laser-induced plasmas," SPIE Proc., 3105, 203-212 (1997).

B. J. Marquardt, D. N. Stratis, D. A. Cremers, and S. M. Angel, "A Novel Probe for Laser-Induced Breakdown Spectroscopy and Raman Measurements Using an Imaging Optical Fiber," Appl. Spectrosc., 42, 1148-1153, (1998).

D. N. Stratis, K. L. Eland, and S. M. Angel, "Dual-Pulse LIBS: Why are two lasers better than one?" SPIE Environ. Monitoring and Remediation Tech., 3853-61, (2000).

K. L. Eland, D. N. Stratis, J. C. Carter and S. M. Angel, "Development of a dual-pulse fiber-optic LIBS probe for in-situ elemental analyses," SPIE Environ. Monitoring and Remediation Tech., 3853-42, (2000).

S. M. Angel, J. C. Carter, D. N. Stratis, B. J. Marquardt, and W. E. Brewer, "Some New Uses for Filtered Fiber-Optic Raman Probes: In Situ Drug Identification and In Situ and Remote Raman Imaging," J. Raman Spectrosc., 30, 795-805, (1999).

D. N. Stratis, and S. M. Angel, "Characterization of Laser-Induced Plasmas for Fiber-Optic Probes," SPIE Environ. Monitoring and Remediation Tech., 3534-88, 592-600, (1999).The Influence of Surface Treatment and Mechanical Properties in the LaserInduced Breakdown Spectroscopy of Copper Samples Allison Oxsher, Lori Grabill, and Scott R. Goode, submitted to J. Anal Atomic Spectrosc.

Identifying Alloys by Laser-Induced Breakdown Spectroscopy with a Time-Resolved High Resolution Spectrometer Scott R. Goode, Stephen L. Morgan, Richard Hoskins, Allison Oxsher, submitted to J. Anal Atomic Spectrosc.

D. N. Stratis, K. L. Eland, and S. M. Angel, "Dual-Pulse LIBS Using a Pre-Ablation Spark for Enhanced Ablation and Emission," Submitted to Appl. Spectrosc., December (1999).

\section{Internet access}

http://ww2.chem.sc.edu/goode/srg/research.htm 